\title{
Correction to: A Theory of Narrative Drawing
}

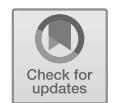

\section{Simon Grennan}

There are typographical errors in this book. The index item "etiology" should read "aetiology". These has been corrected.

The updated online version of the original book can be found at https://doi.org/10.1057/978-1-137-51844-6.

S. Grennan, $A$ Theory of Narrative Drawing, DOI 10.1057/978-1-137-51844-6 5,

(C) The Editor(s) (if applicable) and The Author(s), 2019 